\title{
Cardy-Verlinde formula of Kehagias-Sfetsos black hole
}

\author{
M. R. Setare * \\ Department of Science, Payame Noor University, Bijar, Iran \\ Mubasher Jamil ${ }^{\dagger}$ \\ Center for Advanced Mathematics and Physics, National University of \\ Sciences and Technology, Rawalpindi, 46000, Pakistan
}

August 23, 2018

\begin{abstract}
Abstract: In this paper, we have shown that the entropy of the Kehagias-Sfetsos black hole in Hořava-Lifshitz (HL) gravity can be expressed by the Cardy-Verlinde formula. The later is supposed to be an entropy formula of conformal field theory in any dimension.
\end{abstract}

Keywords: Cardy-Verlinde formula; Kehagias-Sfetsos black hole; Hořava-Lifshitz.

\footnotetext{
*E-mail: rezakord@ipm.ir

†E-mail: mjamil@camp.nust.edu.pk
} 


\section{Introduction}

Verlinde put forward a very interesting formula [1] which relates the entropy of conformal field theory in arbitrary dimension to its total energy and Casimir energy. It has been shown to hold for topological Reissner-Nordström [2] and topological Kerr-Newman [3] black holes in de Sitter spaces, Taub-Bolt- $A d S_{4}$ 4], Kerr-(A)dS [5] and BTZ black hole [6]. There are many other relevant papers on the subject [7, 8, 9]. Thus, one may naively expect that the entropy of all CFTs that have an AdS-dual description is given as the form. However, AdS black holes do not always satisfy the Cardy-Verlinde formula [10]. The aim of this paper is to further investigate the AdS/CFT correspondence in terms of Cardy-Verlinde entropy formula.

Recently, a power-counting renormalizable, ultra-violet (UV) complete theory of gravity was proposed by Hořava in [11, 12, 13, 14. Although presenting an infrared (IR) fixed point, namely General Relativity, in the UV the theory possesses a fixed point with an anisotropic, Lifshitz scaling between time and space. Due to these novel features, there has been a large amount of effort in examining and extending the properties of the theory itself [15, 16, 17, 18, 19, 30, 31, 20, 21, 22, 32, 33, 34, 23, 24, 25, 26, 27, 28, 29. Additionally, application of Hořava-Lifshitz gravity as a cosmological framework gives rise to Hořava-Lifshitz cosmology, which proves to lead to interesting behavior [35, 36, 37. In particular, one can examine specific solution subclasses [38, 39, 40, 41, 42, 43, 44, the phase-space behavior [45, 46], the gravitational wave production [47, 48, 49, 50, 51], the perturbation spectrum [52, 53, 54, 55, 57, 59, 60, 56], the matter bounce [61, 62, 64, 63, the black hole properties [65, 66, 67, 68, 69, 70, 771, 72, 73, 74, 83, the dark energy phenomenology [75, 76, 77], the astrophysical phenomenology [78, 79, 80], the thermodynamic properties [81, 82] etc. However, despite this extended research, there are still many ambiguities if Horava-Lifshitz gravity is reliable and capable of a successful description of the gravitational background of our world, as well as of the cosmological behavior of the universe [19, 30, 21, 23, 84]. In the present paper we would like to check the consistency of the Cardy-Verlinde formula, for the Kehagias-Sfetsos black hole.

\section{Kehagias-Sfetsos black hole}

The natural setting of Hořova-Lifshitz gravity is the ADM formalism, where the four dimensional metric is parameterized by the following

$$
d s^{2}=-N^{2} c^{2} d t^{2}+g_{i j}\left(d x^{i}+N^{i} d t\right)\left(d x^{j}+N^{j} d t\right) .
$$

Here $N$ is the lapse function and $N_{i}$ is the shift function, respectively. The Hořova action is

$$
\begin{aligned}
S= & \int d t d x^{3} \sqrt{g} N\left[\frac{2}{\kappa^{2}}\left(K_{i j} K^{i j}-\lambda_{g} K^{2}\right)-\frac{\kappa^{2}}{2 \nu_{g}^{4}} C_{i j} C^{i j}+\frac{\kappa^{2} \mu}{2 \nu_{g}^{4}} \epsilon^{i j k} R_{i l}^{(3)} \nabla_{j} R_{k}^{(3) l}\right. \\
& -\frac{\kappa^{2} \mu^{2}}{8} R_{i j}^{(3)} R^{(3) i j}+\frac{\kappa^{2} \mu^{2}}{8\left(3 \lambda_{g}-1\right)}\left(\frac{4 \lambda_{g}-1}{4}\left(R^{(3)}\right)^{2}-\Lambda_{W} R^{(3)}+3 \Lambda_{W}^{2}\right) \\
& \left.+\frac{\kappa^{2} \mu^{2} w}{8\left(3 \lambda_{g}-1\right)} R^{(3)}\right],
\end{aligned}
$$


where $\kappa, \lambda_{g}, \nu_{g}, \mu, w$ and $\Lambda_{W}$ are constant parameters. Also $R^{(3)}$ is a 3-dimensional curvature scalar for $g_{i j} ; K_{i j}$ is the extrinsic curvature given by

$$
K_{i j}=\frac{1}{2 N}\left(\dot{g_{i j}}-\nabla_{i} N_{j}-\nabla_{j} N_{i}\right)
$$

is the extrinsic curvature and

$$
C^{i j}=\frac{\epsilon^{i j k}}{\sqrt{g}} \nabla_{k}\left(R_{i}^{j}-\frac{1}{4} R \delta_{i}^{j}\right),
$$

is the Cotton-York tensor. The fundamental constants including speed of light $c$, Newton's gravitational constant $G$ and the cosmological constant $\Lambda$ are defined as

$$
c^{2}=\frac{\kappa^{2} \mu^{2}\left|\Lambda_{W}\right|}{8\left(3 \lambda_{g}-1\right)^{2}}, \quad G=\frac{\kappa^{2} c^{2}}{16 \pi\left(3 \lambda_{g}-1\right)}, \quad \Lambda=\frac{3}{2} \Lambda_{W} c^{2} .
$$

Consider a static and spherically symmetric solution given by

$$
d s^{2}=-e^{\nu(r)} d t^{2}+e^{\lambda(r)} d r^{2}+r^{2}\left(d \theta^{2}+\sin ^{2} d \phi^{2}\right)
$$

By substituting the above metric ansatz into the action, the resulting reduce Lagrangian is given by

$$
\begin{aligned}
\mathbb{L}= & \frac{\kappa^{2} \mu^{2}}{8\left(1-3 \lambda_{g}\right)} e^{(\nu+\lambda) / 2}\left[\left(2 \lambda_{g}-1\right) \frac{\left(e^{-\lambda}-1\right)^{2}}{r^{2}}+2 \lambda_{g} \frac{e^{-\lambda}-1}{r}\left(\lambda^{\prime} e^{-\lambda}\right)+\frac{\lambda_{g}-1}{2}\left(\lambda^{\prime} e^{-\lambda}\right)^{2}\right. \\
& \left.-2\left(w-\Lambda_{W}\right)\left(1-e^{-\lambda}\left(1-r \lambda^{\prime}\right)\right)-3 \Lambda_{W}^{2} r^{2}\right] .
\end{aligned}
$$

The above Lagrangian yields the following equations of motion:

$$
\begin{aligned}
0= & \left(2 \lambda_{g}-1\right) \frac{\left(e^{-\lambda}-1\right)^{2}}{r^{2}}-2 \lambda_{g} \frac{e^{-\lambda}-1}{r}\left(\lambda^{\prime} e^{-\lambda}\right)+\frac{\lambda_{g}-1}{2}\left(\lambda^{\prime} e^{-\lambda}\right)^{2} \\
& -2\left(w-\Lambda_{W}\right)\left[1-e^{-\lambda}\left(1-r \lambda^{\prime}\right)\right]-3 \Lambda_{W}^{2} r^{2} \\
0= & \frac{\nu^{\prime}+\lambda^{\prime}}{2}\left[\left(\lambda_{g}-1\right)\left(\lambda^{\prime} e^{-\lambda}\right)-2 \lambda_{g} \frac{e^{-\lambda}-1}{r}+2\left(w-\Lambda_{W}\right) r\right] \\
& +\left(\lambda_{g}-1\right)\left[\left(-\lambda^{\prime \prime}+\lambda^{\prime}\right) e^{-\lambda}-2 \frac{e^{-\lambda}-1}{r^{2}}\right]
\end{aligned}
$$

by varying functions $\nu$ and $\lambda$ respectively.

Now by imposing $\lambda_{g}=1$, which reduces to the Einstein-Hilbert action in the infra-red limit, one obtains the following solution of the vacuum field equations in Hořava gravity:

$$
e^{\nu(r)}=e^{-\lambda(r)}=1+\left(w-\Lambda_{W}\right) r^{2}-\sqrt{r\left[w\left(w-2 \Lambda_{W}\right) r^{3}+\beta\right]} .
$$

Here $\beta$ is an integration constant. Now the Kehagias-Sfetsos (KS) black hole solution [85] is obtained by considering $\beta=4 w M$ and $\Lambda_{W}=0$,

$$
e^{\nu(r)}=1+w r^{2}-w r^{2} \sqrt{1+\frac{4 M}{w r^{3}}} .
$$


If we impose the limit $\frac{4 M}{w r^{3}} \ll 1$, then the last expression yields the Schwarzschild metric $e^{\nu(r)}=1-2 M / r$. There are two horizons

$$
r_{ \pm}=M\left[1 \pm \sqrt{1-\frac{1}{2 w M^{2}}}\right] .
$$

To avoid a naked singularity at the origin, impose the condition $w M^{2} \gg 1$, the outer horizon approaches the Schwarzschild horizon $r_{+} \simeq 2 M$, and the inner horizon approaches the central singularity, $r_{-} \simeq 0$.

\section{Cardy-Verlinde formula}

In this section, we introduce the Cardy-Verlinde formula which states that the entropy of a $(1+1)$-dimensional CFT is given by

$$
S=2 \pi \sqrt{\frac{c^{\prime}}{6}\left(L_{0}-\frac{c^{\prime}}{24}\right)},
$$

where $c^{\prime}$ is the central charge and $L_{0}$ is the Virasoro generator. After appropriate identifications of $c^{\prime}$ and $L_{0}$, the above Cardy formula, we obtain the generalized Cardy-Verlinde formula which takes the form [1]

$$
S_{C F T}=\frac{2 \pi R}{\sqrt{a b}} \sqrt{E_{C}\left(2 E-E_{C}\right)},
$$

where $E$ is the total energy, $E_{C}$ is the Casimir energy, $a$ and $b$ are arbitrary positive constants. Also $R$ is the radius of the $n+1$ dimensional spacetime, $d s^{2}=-d t^{2}+R^{2} d \Omega_{n}$. The definition of Casimir energy is derived by the violation of the Euler relation as

$$
E_{C}=n(E+P V-T S-\Phi Q-\Omega J),
$$

where the pressure of the CFT is given by $P=E / n V$. For KS black hole, $J$ and $Q$ are zero. The total energy is the sum of two terms

$$
E(S, V)=E_{E}(S, V)+\frac{1}{2} E_{C}(S, V) .
$$

Here $E_{E}$ is the purely extensive part of the total energy. The Casimir energy and the purely extensive part of the total energy are expressed as

$$
\begin{aligned}
& E_{C}=\frac{b}{2 \pi R} S^{1-\frac{1}{n}}, \\
& E_{E}=\frac{a}{4 \pi R} S^{1+\frac{1}{n}} .
\end{aligned}
$$

\section{Entropy of Kehagias-Sfetsos black hole and Cardy- Verlinde formula}

Following [86], the entropy of the KS black hole is assumed to be given by $S=A / 4=\pi r_{+}^{2}$, which yields

$$
S=\pi M^{2}\left[1+\sqrt{1-\frac{1}{2 w M^{2}}}\right]^{2} .
$$


Padmanabhan [87] has shown that temperature of the event horizon of a spherically symmetric spacetime is given by

$$
\begin{aligned}
T & =\frac{1}{4 \pi} \frac{\partial e^{\nu(r)}}{\partial r}, \\
& =\frac{w}{2 \pi}\left[r_{+}-r_{+} \sqrt{1+\frac{4 M}{w r_{+}^{3}}}+\frac{3 M}{r_{+}^{2} \sqrt{1+\frac{4 M}{w r_{+}^{3}}}}\right]
\end{aligned}
$$

calculated at the event horizon $r=r_{+}$. We choose $n=2$ and $E=M$ for KS black hole. The Casimir energy becomes

$$
\begin{aligned}
E_{C} & =3 M-2 T S \\
& =3 M-w M^{2}\left[r_{+}-r_{+} \sqrt{1+\frac{4 M}{w r_{+}^{3}}}+\frac{3 M}{r_{+}^{2} \sqrt{1+\frac{4 M}{w r_{+}^{3}}}}\right]\left[1+\sqrt{1-\frac{1}{2 w M^{2}}}\right]^{2} .
\end{aligned}
$$

The pure extensive part of the total energy is given by

$$
\begin{aligned}
E_{E} & =-\frac{1}{2} M+T S \\
& =-\frac{1}{2} M+\frac{w M^{2}}{2}\left[r_{+}-r_{+} \sqrt{1+\frac{4 M}{w r_{+}^{3}}}+\frac{3 M}{r_{+}^{2} \sqrt{1+\frac{4 M}{w r_{+}^{3}}}}\right]\left[1+\sqrt{1-\frac{1}{2 w M^{2}}}\right]^{2} .
\end{aligned}
$$

Also

$$
\begin{aligned}
2 E-E_{C} & =-M+2 T S \\
& =-M+w M^{2}\left[r_{+}-r_{+} \sqrt{1+\frac{4 M}{w r_{+}^{3}}}+\frac{3 M}{r_{+}^{2} \sqrt{1+\frac{4 M}{w r_{+}^{3}}}}\right]\left[1+\sqrt{1-\frac{1}{2 w M^{2}}}\right]^{2} .
\end{aligned}
$$

From comparison of equations (17) and (21), we obtain

$$
\begin{aligned}
R= & \frac{b S^{1 / 2}}{4 \pi}\left(\frac{3}{2} M-T S\right)^{-1} \\
= & \frac{b}{4 \pi^{1 / 2}} M\left(1+\sqrt{1-\frac{1}{2 w M^{2}}}\right)\left[\frac{3}{2} M-\frac{w M^{2}}{2}\left(r_{+}-r_{+} \sqrt{1+\frac{4 M}{w r_{+}^{3}}}+\frac{3 M}{r_{+}^{2} \sqrt{1+\frac{4 M}{w r_{+}^{3}}}}\right)\right. \\
& \left.\times\left(1+\sqrt{1-\frac{1}{2 w M^{2}}}\right)^{2}\right]^{-1}
\end{aligned}
$$

From comparison of equations (18) and (22), we obtain

$R=\frac{a S^{3 / 2}}{4 \pi}\left(-\frac{1}{2} M+T S\right)^{-1}$, 


$$
\begin{aligned}
= & \frac{a}{4} \pi^{1 / 2} M^{3}\left(1+\sqrt{1-\frac{1}{2 w M^{2}}}\right)^{3}\left[-\frac{1}{2} M+\frac{w M^{2}}{2}\left(r_{+}-r_{+} \sqrt{1+\frac{4 M}{w r_{+}^{3}}}+\frac{3 M}{r_{+}^{2} \sqrt{1+\frac{4 M}{w r_{+}^{3}}}}\right)\right. \\
& \left.\times\left(1+\sqrt{1-\frac{1}{2 w M^{2}}}\right)^{2}\right]^{-1} .
\end{aligned}
$$

Taking product of (24) and (25), we obtain

$$
R=\frac{\sqrt{a b}}{4 \pi} \frac{S}{\sqrt{\left(\frac{3}{2} M-T S\right)^{-1}\left(-\frac{1}{2} M+T S\right)^{-1}}}
$$

Using (21), (22) and (26) in (14), we obtain

$$
S_{C F T}=S
$$

It has been proven that the entropy of the Kehagias-Sfetsos black hole can be expressed in the form of Cardy-Verlinde formula. We would like to remark that the technique used above to prove this result has been followed earlier for the rotating charged BTZ black hole as well [6, 8]. Moreover, the quantity $R$ is in general arbitrary but in the presence of a background geometry, it becomes particular and therefore can be written in terms of the parameters of Kehagias-Sfetsos spacetime (the geometry), like we obtained in (26).

\section{Conclusion}

HL theory brings some important new features from the GR to the higher dimensional lagrangian and it's role in construction a non relativistic candidate for quantum gravity. According to the Blas et al arguments [26], it seems that this model must be modified by some terms to avoiding from strong coupling, instabilities, dynamical in consistencies and unphysical extra mode. One of the first exact solutions for this modified version is the work of Kiritsis [88. Indeed the Kiritsis work contains some previous families of exact solutions as a special sub class and has a good asymptotic behaviors. The explicit form of exact solution for this modified version deal with some algebraic quadratures and lead finally to an implicit static spherically symmetric metric. But no doubt this solution generic, avoids from the trouble problems which occur in the original version of HL. As we know that there are 2 explicit family of exact solutions for a spherically symmetric background without projectability condition and other solutions all are the familiar GR solution i.e $A d s_{4}$-Schwarzchild solutions. One solution belongs to the [85] which in abberation in literatures known as KS solution. This solution is asymptotically flat and as we showed that in spite of the GR BHs, it's timelike geodesics is stable [89]. The aim of this paper is to further investigate the AdS/CFT correspondence in terms of Cardy-Verlinde entropy formula. We have shown that the entropy of the black hole horizon of Kehagias-Sfetsos spacetime can also be written in the form of Cardy-Verlinde entropy formula. 


\section{References}

[1] E. Verlinde, hep-th/0008140.

[2] M. R. Setare, Mod. Phys. Lett. A 17, 2089, (2002).

[3] M. R. Setare, and M. B. Altaie, Eur. Phys. J. C 30, 273, (2003).

[4] D. Birmingham and S. Mokhtari, Phys. Lett. B 508, 365 (2001); Ch. O. Lee, Phys. Lett. B670, 146, (2008).

[5] D. Klemm, A. C. Petkou and G. Siopsis, Nucl. Phys. B 601, 380 (2001).

[6] M.R. Setare and M. Jamil, Phys. Lett. B 681 (2009) 471.

[7] M. R. Setare and R. Mansouri, Int. J. Mod. Phys. A 18, 4443 (2003).

[8] M. R. Setare and E. C. Vagenas, Phys. Rev. D 68 (2003) 064014; M. R. Setare and E. C. Vagenas, Int. J. Mod. Phys. A 20, 7219, (2005); M. R. Setare, Eur.Phys.J. C33, 555 (2004).

[9] B. Wang, E. Abdalla and R. K. Su, Phys. Lett. B 503, 394 (2001).

[10] G. W. Gibbons, M. J. Perry and C. N. Pope, Phys. Rev. D 72, 084028 (2005).

[11] P. Horava, arXiv:0811.2217 [hep-th].

[12] P. Horava, JHEP 0903, 020 (2009) arXiv:0812.4287 [hep-th]].

[13] P. Horava, Phys. Rev. D 79, 084008 (2009) [arXiv:0901.3775 [hep-th]].

[14] P. Hořava, arXiv:0902.3657 [hep-th].

[15] G. E. Volovik, arXiv:0904.4113 [gr-qc].

[16] D. Orlando and S. Reffert, arXiv:0905.0301 [hep-th].

[17] T. Nishioka, arXiv:0905.0473 [hep-th].

[18] R. A. Konoplya, arXiv:0905.1523 [hep-th].

[19] C. Charmousis, G. Niz, A. Padilla and P. M. Saffin, arXiv:0905.2579 [hep-th].

[20] T. P. Sotiriou, M. Visser and S. Weinfurtner, Phys. Rev. Lett. 102, 251601 (2009) arXiv:0904.4464 [hep-th]].

[21] T. P. Sotiriou, M. Visser and S. Weinfurtner, JHEP 0910, 033 (2009) arXiv:0905.2798 [hep-th]].

[22] C. Germani, A. Kehagias and K. Sfetsos, JHEP 0909, 060 (2009) arXiv:0906.1201 [hep-th]].

[23] C. Bogdanos and E. N. Saridakis, arXiv:0907.1636 [hep-th].

[24] J. Kluson, arXiv:0907.3566 [hep-th]. 
[25] J. Alexandre, K. Farakos, P. Pasipoularides and A. Tsapalis, arXiv:0909.3719 [hepth].

[26] D. Blas, O. Pujolas and S. Sibiryakov, arXiv:0909.3525 [hep-th].

[27] D. Capasso and A. P. Polychronakos, arXiv:0909.5405 [hep-th].

[28] B. Chen, S. Pi and J. Z. Tang, arXiv:0910.0338 [hep-th].

[29] J. Kluson, arXiv:0910.5852 [hep-th].

[30] M. Li and Y. Pang, arXiv:0905.2751 [hep-th].

[31] M. Visser, arXiv:0902.0590 [hep-th].

[32] J. Chen and Y. Wang, arXiv:0905.2786 [gr-qc].

[33] B. Chen and Q. G. Huang, arXiv:0904.4565 [hep-th].

[34] F. W. Shu and Y. S. Wu, arXiv:0906.1645 [hep-th].

[35] G. Calcagni, arXiv:0904.0829 [hep-th].

[36] E. Kiritsis and G. Kofinas, arXiv:0904.1334 [hep-th].

[37] R. G. Cai, B. Hu and H. B. Zhang, Phys. Rev. D 80, 041501 (2009).

[38] H. Lu, J. Mei and C. N. Pope, arXiv:0904.1595 [hep-th].

[39] H. Nastase, arXiv:0904.3604 [hep-th].

[40] M. Minamitsuji, arXiv:0905.3892 [astro-ph.CO].

[41] P. Wu and H. W. Yu, arXiv:0909.2821 [gr-qc].

[42] I. Cho and G. Kang, arXiv:0909.3065 [hep-th].

[43] C. G. Boehmer and F. S. N. Lobo, arXiv:0909.3986 [gr-qc].

[44] M. R. Setare, D. Momeni, arXiv:0911.1877v2 [hep-th].

[45] S. Carloni, E. Elizalde and P. J. Silva, arXiv:0909.2219 [hep-th].

[46] G. Leon and E. N. Saridakis, arXiv:0909.3571 [hep-th].

[47] S. Mukohyama, K. Nakayama, F. Takahashi and S. Yokoyama, arXiv:0905.0055 [hepth].

[48] T. Takahashi and J. Soda, arXiv:0904.0554 [hep-th].

[49] S. Koh, arXiv:0907.0850 [hep-th].

[50] M. i. Park, arXiv:0910.1917 [hep-th].

[51] M. i. Park, arXiv:0910.5117 [hep-th]. 
[52] S. Mukohyama, arXiv:0904.2190 [hep-th].

[53] Y. S. Piao, arXiv:0904.4117 [hep-th].

[54] X. Gao, arXiv:0904.4187 [hep-th].

[55] B. Chen, S. Pi and J. Z. Tang, arXiv:0905.2300 [hep-th].

[56] Ch. Ding, S. Chen, J. Jing, Phys.Rev. D 81, (2010) 024028.

[57] X. Gao, Y. Wang, R. Brandenberger and A. Riotto, arXiv:0905.3821 [hep-th].

[58] A. Wang and R. Maartens, arXiv:0907.1748 [hep-th].

[59] T. Kobayashi, Y. Urakawa and M. Yamaguchi, arXiv:0908.1005 [astro-ph.CO].

[60] A. Wang, D. Wands and R. Maartens, arXiv:0909.5167 [hep-th].

[61] R. Brandenberger, arXiv:0904.2835 [hep-th].

[62] R. H. Brandenberger, arXiv:0905.1514 [hep-th].

[63] Y. F. Cai and E. N. Saridakis, JCAP 0910, 020 (2009) [arXiv:0906.1789 [hep-th]].

[64] T. Suyama, arXiv:0909.4833 [hep-th].

[65] U. H. Danielsson and L. Thorlacius, JHEP 0903, 070 (2009) arXiv:0812.5088 [hepth]].

[66] R. G. Cai, L. M. Cao and N. Ohta, arXiv:0904.3670 [hep-th].

[67] A. Kehagias and K. Sfetsos, Phys. Lett. B 678, 123 (2009) [arXiv:0905.0477 [hep-th]].

[68] R. B. Mann, arXiv:0905.1136 [hep-th].

[69] G. Bertoldi, B. A. Burrington and A. Peet, arXiv:0905.3183 [hep-th].

[70] M. i. Park, JHEP 0909, 123 (2009) [arXiv:0905.4480 [hep-th]].

[71] A. Castillo and A. Larranaga, arXiv:0906.4380 [gr-qc].

[72] M. Botta-Cantcheff, N. Grandi and M. Sturla, arXiv:0906.0582 [hep-th].

[73] N. Varghese and V. C. Kuriakose, arXiv:0909.4944 [gr-qc].

[74] E. Kiritsis and G. Kofinas, arXiv:0910.5487 [hep-th].

[75] E. N. Saridakis, arXiv:0905.3532 [hep-th].

[76] C. Appignani, R. Casadio and S. Shankaranarayanan, arXiv:0907.3121 [hep-th].

[77] M. R. Setare, arXiv:0909.0456 [hep-th]; M. R. Setare, Mubasher Jamil, arXiv:1001.1251v1 [hep-th], accepted for publication in JCAP (2010).

[78] S. S. Kim, T. Kim and Y. Kim, arXiv:0907.3093 [hep-th]. 
[79] T. Harko, Z. Kovacs and F. S. N. Lobo, arXiv:0908.2874 [gr-qc].

[80] L. Iorio and M. L. Ruggiero, arXiv:0909.5355 [gr-qc].

[81] A. Wang and Y. Wu, arXiv:0905.4117 [hep-th].

[82] R. G. Cai, L. M. Cao and N. Ohta, arXiv:0905.0751 [hep-th].

[83] B. R. Majhi, arXiv:0911.3239v1 [hep-th].

[84] K. Koyama and F. Arroja, arXiv:0910.1998 [hep-th].

[85] A. Kehagias and K. Sfetsos, Phys. Lett. B678, 123 (2009); F. S. N. Lobo et al, arXiv:1001.3517v1 [gr-qc]

[86] Q-J Cao et al, arXiv:1001.2597 [hep-th]

[87] T. Padmanabhan, arXiv:gr-qc/0204019v2.

[88] E.Kiritsis, "Spherically symmetric solutions in modified Hořava-Lifshitz gravity," arXiv:0911.3164 [gr-qc]].

[89] M. R. Setare, D. Momeni, "Geodesic stability for KS Black hole in Hořava-Lifshitz gravity via Lyapunov exponents ", arXiv:1001.3767v1 [physics.gen-ph]. 